\title{
Editors' acknowledgements
}

The Editors are deeply grateful to the following individuals, who contributed to the review process by submitting comments on one or more manuscripts.

Special thanks are due to those, who offered also their linguistic help.

J. R. Adams, R. M. Adkins, G. Amori, H. Andrén, K. D. Angielczyk, H. Ansorge, B. M. Barnes, G. O. Batzli, R. M. Baxter, F. W. Bazer, A. Berta, C. Bishop, L. Bonesi, P. M. Borodin, Z. Borowski, L. F. C. Brito, B. W. Brook, M. Brzeziński, H. Burda, D. Capizzi, R. Castiglia, N. G. Chapman, S. Churchfield, P. B. Conn, T. Cornulier, T. R. O. de Freitas, S. de Solla, S. M. Dloniak, S. Erlinge, S. H. Ferguson, R. J. Fisher, J. R. Flowerdew, S. Focardi, B. J. Fox, K. Fredga, B. Galef Jr, S. D. Gehrt, L. L. Getz, W. M. Getz, G. Gheusi, J. Gliwicz, T. E. Gosselink, A. Goswami, K. Gough, L. A. Harrington, L. D. Hayes, M. W. Hayward, A. M. Henderson, J. Herrero, D. J. Hosken, N. H. Ing, J. L. Isaac, J. Jacob, J. Jacquot, A. J. T.
Johnsingh, R. Juškaitis, K. Kauhala, C. M. King, R. W. Klaver, I. Klemme, R. Klenke, M. Konarzewski, R. Kowalczyk, F. J. L. Kraaijeveld-Smit, B. Kryštufek, R. Kühn, J. Lanszki, N. O. Leiner, E. Lemazurier, M. Lima, J. D. C. Linnell, M. F. E. Loughran, S. Lovari, J. Lynch Alfaro, M. Macholán, S. B. Magle, G. Massei Smith, C. Melis, A. Meriggi, J. S. Millar, A. Mira, A. J. Mitchell-Jones, O. B. Mock, I. T. Moore, D. W. Morris, A. Mortelliti, B. W. Moser, M. E. Moses, E. Möstl, J. Munshi-South, J. O. Murie, A.-M. Mustonen, C. K. Nielsen, A. Novello, V. A. Olson, A. Orihuela, R. Palme, J. D. Perrine, N. Pettorelli, F. Pezzo, N. Pillay, J. Pluháček, P. D. Polly, K. G. Poole, J. H. Porter, P. A. Racey, S. A. Ramm, J.-F. Robitaille, S. Rommel, R. K. Rose, I. Ruczyński, R. Rutkowski, B. N. Sacks, A. I. Schulte-Hostedde, N. A. Slade, N. G. Solomon, P. Soppela, R. Šumbera, J. R. E. Taylor, C. Tod'go, A. Toledano Díaz, I. Torre, W. C. Turner, E. M. Vieira, C. G. Vlachos, F. Vollrath, W. Walankiewicz, E. L. Walters, J.-M. Weber, T. A. White, J. G. White, M. Wolsan, J. Woodruff, A. Xu, N. Yamaguchi, R. Young, R. R. Zenuto, J. Zima. 